\title{
Observer weighting of interaural delays in filtered impulses
}

\author{
KOUROSH SABERI \\ University of Florida, Gainesville, Florida
}

\begin{abstract}
Onset dominance in sound localization was examined by estimating observer weighting of interaural delays for each click of a train of high-frequency filtered clicks. The interaural delay of each click was a normal deviate that was sampled independently on each trial of a single-interval design. In Experiment 1 , observer weights were derived for trains of $n=2,4,8$, or 16 clicks as a function of interclick interval (ICI $=1.8,3.0$, or $12.0 \mathrm{msec}$ ). For small $n$ and short ICI $(1.8 \mathrm{msec})$, the ratio of onset weight to remaining weights was as large as 10 . As ICI increased, the relative onset weight was reduced. For large $n$ and all ICIs, the ongoing train was weighted more heavily than the onset. This diminishing relative onset weight with increasing ICI and $n$ is consistent with optimum distribution of weights among components. Efficiency of weight distribution is near ideal when ICI $=12 \mathrm{msec}$ and $n=$ 2 and very poor for shorter ICIs and larger $n$ s. Further experiments showed that: (1) onset dominance involves both within and between-frequency-channel mechanisms, and (2) the stimulus configuration (ICI, $n$, frequency content, and temporal gaps) affects weighting functions in a complex way not explained by cross-correlation analysis or contralateral inhibition (Lindemann, 1986a, 1986b).
\end{abstract}

The earliest empirical documentation of onset dominance in sound localization dates to the mid-nineteenth century (Henry, 1849/1851). Exactly 100 years later, the systematic study of this phenomenon began with two independently published papers by Haas (1949/1972) in Germany and Wallach, Newman, and Rosenzweig (1949) in the United States. Haas coined the phrase $a u$ ditory-suppression effect and Wallach et al., the term precedence effect (more widely used in the psychoacoustic community), both referring to the dominance of stimulus-onset information in determining the perceived spatial location of an acoustic stimulus. Since publication of these papers, the precedence effect has been the topic of extensive theoretical (Lindemann, 1986a, 1986b; Zurek, 1987), physiological (Cranford \& Oberholtzer, 1976; Litovsky, 1994; Yin \& Litovsky, 1994), applied (Blauert, 1989; Muncey, Nickson, \& Dubout, 1953), and even clinical (Hochster \& Kelly, 1981) study within the hearing sciences.

In spite of this extended history, the mechanisms that underlie the precedence effect are not well understood. Recent views consider the precedence effect to include a variety of onset-dominance phenomena that may involve different mechanisms. Some onset effects, such as binaural adaptation (Hafter \& Dye, 1983), are speculated to involve nuclei at relatively early stages of the auditory

This work was supported by NIH and AFOSR. I thank David M. Green, Bruce G. Berg, P. M. Zurek, Wes Grantham, and an anonymous reviewer for many helpful comments. I also thank Zekiye Onsan, Quang Nguyen, and Mary Fullerton for their help throughout this research. The author's present address is Research Laboratory of Electronics 36-765, Massachusetts Institute of Technology, Cambridge, MA 02139 (e-mail: kourosh@mit.edu). tract. For the impulsive stimuli used to demonstrate binaural adaptation and similar effects (Gaskell, 1983; Wallach et al., 1949), onset dominance is most intense when secondary clicks occur within 1 to 3 msec after the onset click. Both psychophysical (Hafter, Buell, \& Richards, 1988 ) and physiological evidence (Litovsky, 1994; Yin \& Litovsky, 1994) suggest that this type of loss in postonset information may occur at or below the level of the inferior colliculus (IC).

Other onset phenomena, also referred to as precedence, seem to be based in much higher levels of the auditory system and may involve cognitive processes or factors related to attention. For example, for complex stimuli such as speech and music signals, precedence is observed for delays of $30 \mathrm{msec}$ or more between the primary wavefront and its simulated "echo" (Haas, 1949/1972)-an exceptionally long delay compared with what is optimum for impulsive sounds or noise waveforms ( $1-3 \mathrm{msec}$; Haas, 1949/1972; Wallach et al., 1949). In some experimental situations, the onset dominance may be influenced by even longer time constants, in the order of several seconds (Clifton, 1987). See Blauert and Col (1991) for a review of irregularities in defining precedence.

Many methods have been used to study onset dominance. Earlier reports used stimuli whose onset interaural information was controlled independently from that in its ongoing part (Aoki \& Houtgast, 1992; Gaskell, 1983; Perrott, Marlborough, Merrill, \& Strybel, 1989; Saberi \& Perrott, 1990; Tobias \& Schubert, 1959; Wallach et al., 1949; Zurek, 1980). To measure onset dominance, for example, the early part of the stimulus was diotic while the ongoing part carried an interaural delay that favored one ear. Lateralization thresholds were then estimated for the later-arriving part and compared with those from the early 
part. Other techniques have included the method of subtraction in which performance with $n$ clicks is compared with performance with $n-k$ clicks (Hafter et al., 1988; Hafter \& Dye, 1983), or trading methods where the onset interaural delay is balanced against that in the trailing part to produce an intracranially centered image (Snow, 1954; Wallach et al., 1949; Yost \& Soderquist, 1984). Nearly all of these studies have shown that the onset is 5 to 10 times as dominant (defined by the relative magnitude of their respective thresholds) as the ongoing stimulus.

In more recent years, complex analysis techniques have been used in the study of onset effects (Houtgast \& Aoki, 1994; Litovsky \& Macmillan, 1994; Shinn-Cunningham, Zurek, \& Durlach, 1993). Shinn-Cunningham et al. proposed a model that incorporated a single parameter for quantifying onset dominance. The model assigned two weights, one to the onset $(c)$ and one to the ongoing interaural delay $(1-c)$. The use of the parameter $c$, bounded by 0 and unity, was based on the assumption of a linear trade between onset and ongoing dominance in lateralization. This single-image, single-parameter model has been shown to produce reasonable fits to data from several studies (Gaskell, 1983; Saberi \& Perrott, 1990; Zurek, 1980 ) and has since been applied to free-field localization (Litovsky \& Macmillan, 1994). In Houtgast and Aoki's technique, although the total duration of the stimulus (noise burst) was fixed, trial-by-trial variability ( 1 of 10 discrete values) was introduced in the duration of the ongoing stimulus. From observers' responses, they quantified a stimulus weighting function. A critical assumption of the Houtgast and Aoki method, as they acknowledge, is that weighting functions are independent of signal duration, that is, the weight at time $t \mathrm{sec}$ after onset is unaffected by the presence or absence of a stimulus at time $t+\varepsilon$. As shown later in this paper, however, some signal parameters produce weighting functions that violate this assumption.

The present study uses a procedure that provides weight estimates for each component of a multicomponent signal (Berg, 1989); the stimulus here is a train of dichotic clicks whose interaural delays are independent normal variables sampled individually for each click on each observation. An appealing feature of this method, as compared with the customary two-weight estimate for onset and ongoing information, is its ability to handle more than two components at a time. One can, for example, estimate the use that an observer makes of spatial information in each and every click of a train. While click trains are used in this study, the method may, in principle, be extended to continuous waveforms if the interaural information at different segments of the waveform may be controlled independently. The method thus allows one to examine such questions as the effect of signal duration on the form of the weighting functions, spectral effects on onset dominance, and the effects of gaps of various durations within the same train.

\section{GENERAL METHOD}

The stimuli were trains of dichotic clicks. Each click was the impulse response of a Gaussian filter with a 1-msec effective temporal envelope (between terminal $0 \mathrm{~V}$ ); the envelope was centered on the cosine phase of a $6-\mathrm{kHz}$ carrier. A single click waveform can be described as

$$
X(t)=\frac{1}{\sigma \sqrt{2 \pi}} e^{-0.5[(t-T / 2) / \sigma]^{2}} \cos [\omega(t-T / 2)]
$$

where $T=10^{-3} \mathrm{sec}$ and $\sigma=1.5\left(10^{-4}\right) \mathrm{sec}$. The amplitude and power spectra of each pulse were also Gaussian with widths within $1 \sigma$ point of the carrier equal to 2800 and $1978 \mathrm{~Hz}$, respectively. Signals were generated in an IBM PC and presented through digital-toanalog converters (TDT DA2) and Sennheiser (HD 450) headphones at a sampling rate of $50 \mathrm{kHz}$. They were lowpass filtered at $20 \mathrm{kHz}(\mathrm{Kemo} \mathrm{VBF} / 24)$. The level of a continuous train $(100 / \mathrm{sec})$ was calibrated to $60 \mathrm{~dB}$ SPL using a digital rms voltmeter (Fluke $8050 \mathrm{~A})$.

\section{Procedure}

A single click train was presented during the visually marked interval of a single-interval, forced-choice design. The number of clicks within the train, $n$, and the interclick interval (ICI) were parameters of the study. The ICI was jittered by $10 \%$ on each trial to reduce monaural pitch cues. Otherwise, both parameters (ICI and $n$ ) were held constant during a run, which consisted of 100 trials. Subjects could practice on each run for an unlimited number of trials, although practice trials usually did not exceed 10 . Before data collection began, the subjects practiced the various conditions of the task until their performance seemed to be stable. Each day, all conditions of a given experiment were run in a randomized order.

Berg's (1989) method was used to determine the weight given to each click of the train. The interaural time difference (ITD) of each click was independently sampled from either a Gaussian distribution $N_{\mathrm{L}} \sim \operatorname{normal}\left(-\mu, \sigma^{2}\right)$ or $N_{\mathrm{R}} \sim \operatorname{normal}\left(\mu, \sigma^{2}\right)$, where negative ITDs represent those interaural delays for which the left ear was leading in time and positive ITDs represent those for which the right ear was leading. On each presentation of the train, the ITDs of all clicks were sampled from only one of these two distributions. The subject's task was to determine from which of the two distributions the train was actually sampled. While the value of $\sigma$ was always $100 \mu \mathrm{sec}$, the value of $\mu$ (distribution mean) was adjusted for each subject and each condition individually to obtain approximately $75 \%$ correct performance. The means, $\mu$, of each distribution were at most about $200 \mu \mathrm{sec}$ leading to one side, and therefore the maximum value of naturally occurring ITDs $\left(650 \mu \mathrm{sec}\right.$ at $90^{\circ}$ azimuth) was $4.5 \sigma$ away from $\mu$. Thus, virtually no trials occurred on which the interaural delay of a given click exceeded $650 \mu \mathrm{sec}$. At the beginning of every run, a diotic sample of the signal for that run was presented over the headphones and repeated at a rate of twice per second. Subjects were instructed to center the image as best as they could by adjusting the positioning of the headphones. This was done to reduce the effects of acoustic leakage caused by asymmetries in the listener's head or by headphone placement (Domnitz, 1973).

To better understand the statistical procedure, assume that on a given trial, distribution $N_{\mathrm{L}}$ is selected. On this trial, the interaural delay of each click $\left(\mathrm{ITD}_{i}\right)$ is sampled from $N_{\mathrm{L}} \sim \operatorname{normal}\left(-\mu, \sigma^{2}\right)$. This is the distribution of interaural delays whose mean is leading toward the left ear (as shown in Figure 1). In this example, if $N_{\mathrm{L}} \sim$ normal $\left(-200,100^{2}\right)$ is the distribution used, we may have $\mathrm{ITD}_{1}=-183, \mathrm{ITD}_{2}=-108, \mathrm{ITD}_{3}=-330, \mathrm{ITD}_{4}=+28 \mu \mathrm{sec}$, and so forth. 


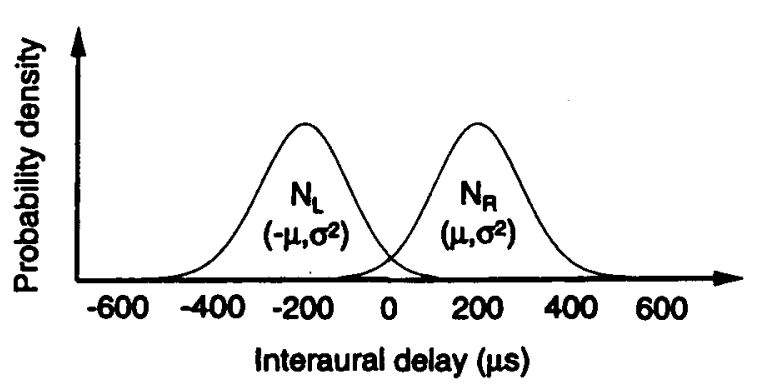

Figure 1. Distribution of interaural delays. Positive delays lead to the right ear; negative values lead to the left ear. Zero interaural delay represents a diotic click. On each trial, one of the two distributions was selected and interaural delay for each click was sampled independently from that distribution.

Let $\mathrm{ITD}_{i}$ represent the interaural delay of the $i$ th click. The method assumes that a weight, $a_{i}$, is given to each click. The weights, $a_{i}$, may be thought of as representing an internal process, either one that the observer may control, for example, by directed attention, or one that may be out of the observer's control, for example, loss of neural samples. It is assumed that a decision statistic, $D$, is formed from the weighted sum of ITD , each corrupted by additive internal noise, $\varepsilon_{i} \sim$ normal $\left(0, \sigma_{\varepsilon_{i}}^{2}\right)$. The decision variable is then compared with criterion $C$ to arrive at a decision:

$$
D=n_{R} \text { iff } \sum_{\text {All } i} a_{i}\left(\operatorname{ITD}_{i}+\varepsilon_{i}\right)>C ; n_{\mathrm{L}} \text { otherwise, }
$$

where $n_{\mathrm{R}}$ and $n_{\mathrm{L}}$ correspond to the two possible states $N_{\mathrm{R}}$ and $N_{\mathrm{L}}$. From this inequality, the relative weights given to arbitrary clicks $j$ and $k$ are derived,

$$
\frac{a_{j}^{2}}{a_{k}^{2}}=\frac{\operatorname{var}\left[Y_{k}\right]+\sigma_{k}^{2}}{\operatorname{var}\left[Y_{j}\right]+\sigma_{j}^{2}},
$$

where $\sigma_{i}$ and $\sigma_{k}$ are standard deviations of the normal distributions from which the interaural delays are sampled and $\operatorname{var}\left[Y_{j}\right]$ and $\operatorname{var}\left[Y_{k}\right]$ are the experimentally determined variances of what Berg refers to as COSS (conditional on single stimulus) functions. Each click is associated with its own COSS function. A COSS function is simply the psychometric function for the observer's performance given stimulus values for a particular click, $j$.

To estimate $\operatorname{var}\left[Y_{j}\right]$, consider the curves depicted in panel $\mathrm{A}$ of Figure 2. Panel B will be discussed shortly, but for now, it should be mentioned that the data of this figure were collected using a 2-click train (an onset click and only a single secondary click). The data of panel $A$ are associated with the 1st click, and the data of panel $B$, with the 2 nd click. These data are partial results from the experiments described below and are shown here to facilitate understanding of the procedure. The lower curve in panel A summarizes the performance of a subject on trials in which distribution $N_{\mathrm{L}} \sim \operatorname{normal}\left(-160,100^{2}\right)$ was used. The upper curve in panel $\mathrm{A}$ summarizes performance on trials in which $N_{\mathrm{R}} \sim \operatorname{normal}\left(160,100^{2}\right)$ was used. On any given trial, either distribution may have been selected. On each trial, we recorded both the ITD of each click of the train and the subject's response given those ITDs. Thus, on each trial, we recorded two values of ITD and one response. ITDs were then grouped into bins to summarize the observer's performance. Here, 16 bins were created (resulting in 16 points per curve) by taking equal areas $(6.25 \%)$ under the normal densities that defined $N_{\mathrm{L}}$ or $N_{\mathrm{R}}$. The probability of responding right, $n_{\mathrm{R}}$, conditioned on distribution $N_{\mathrm{R}}$ and bin number was transformed into $z$ units and, together with a linear least square fit (solid line), was plotted in panel A. The square of the inverse of the slopes $\left(m^{-2}\right)$ of these fits provides an estimate of $\operatorname{var}\left[Y_{j}\right]$. We assume that $\operatorname{var}\left[Y_{j} \mid N_{\mathrm{L}}\right]=\operatorname{var}\left[Y_{j} \mid N_{\mathrm{R}}\right]$, and therefore take the average of the two estimates as the final estimate of $\operatorname{var}\left[Y_{j}\right]$.

Panel B shows performance for the ITD of the 2 nd click. The difference in the slopes of the fits between the two panels is a quantitative measure of the effect of each click on a subject's performance. Panel A of Figure 2 shows that increasing the interaural delay of the 1 st click systematically affected performance, whereas panel B shows that the subject's performance was less dependent on changes in interaural delay. This difference in performance may be quantified in terms of relative weights, $a_{j}$. To obtain these weights from Equation 2, $a_{j=1}$ may be set to unity and the remaining weight derived from the iterative substitution of $k=2, \ldots, n$. As a final step, the sum of the weights may be normalized to unity $\left(\sum a_{i}=1\right){ }^{1}$

\section{EXPERIMENT 1 \\ Weights Derived for \\ Each Click of a Train of $\boldsymbol{n}$ Clicks}

\section{Method}

Weights were determined for each click of a train as a function of the number of clicks in that train $(n=2,4,8$, or 16$)$. Three ICIs were used $(1.8,3$, and $12 \mathrm{msec})$. From previous work in this area (Gaskell, 1983; Hafter et al., 1988; Saberi \& Perrott, 1990; ShinnCunningham et al., 1993; Yost \& Soderquist, 1984), we expected to observe large weights given to the 1 st click for ICIs of 1.8 and $3 \mathrm{msec}$, but not for an ICI of $12 \mathrm{msec}$.
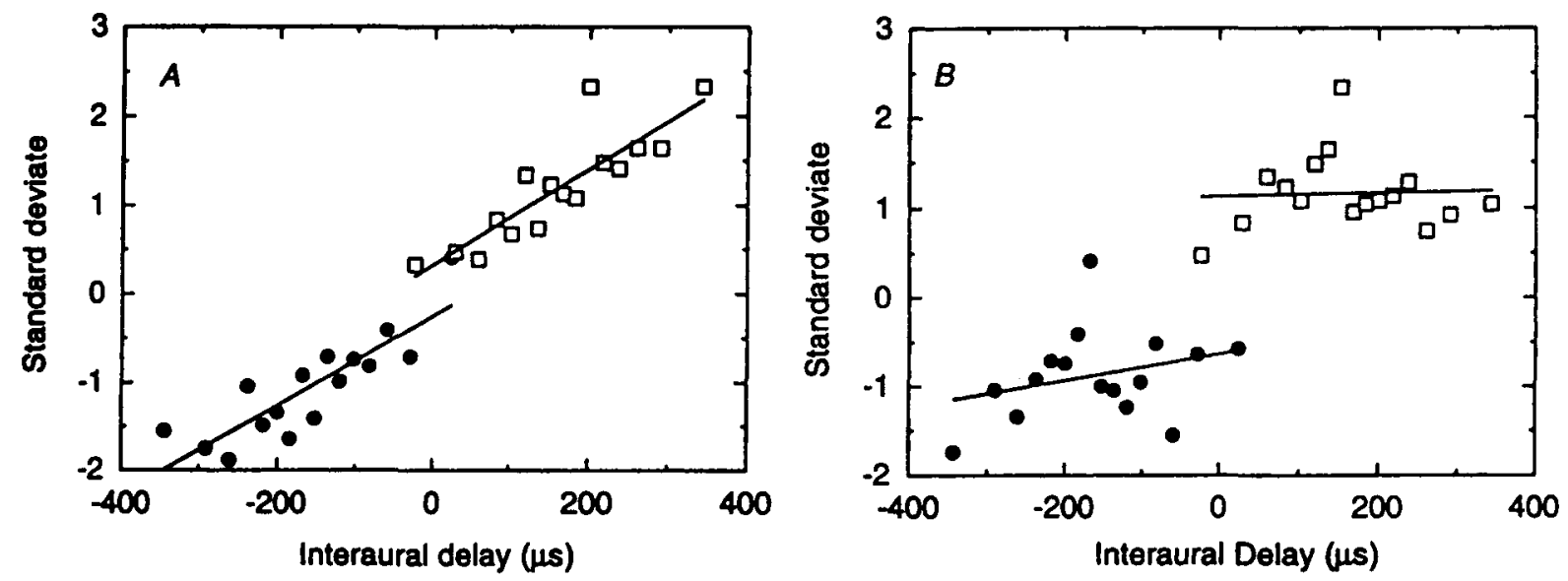

Figure 2. Left and right panels show COSS functions for the 1st and 2nd click, respectively, of a 2-click train obtained for 1 subject (Y.B.). The deviates are from $P\left(n_{R} \mid N_{L}\right)$ for filled symbols and $P\left(n_{R} \mid N_{R}\right)$ for open symbols. 


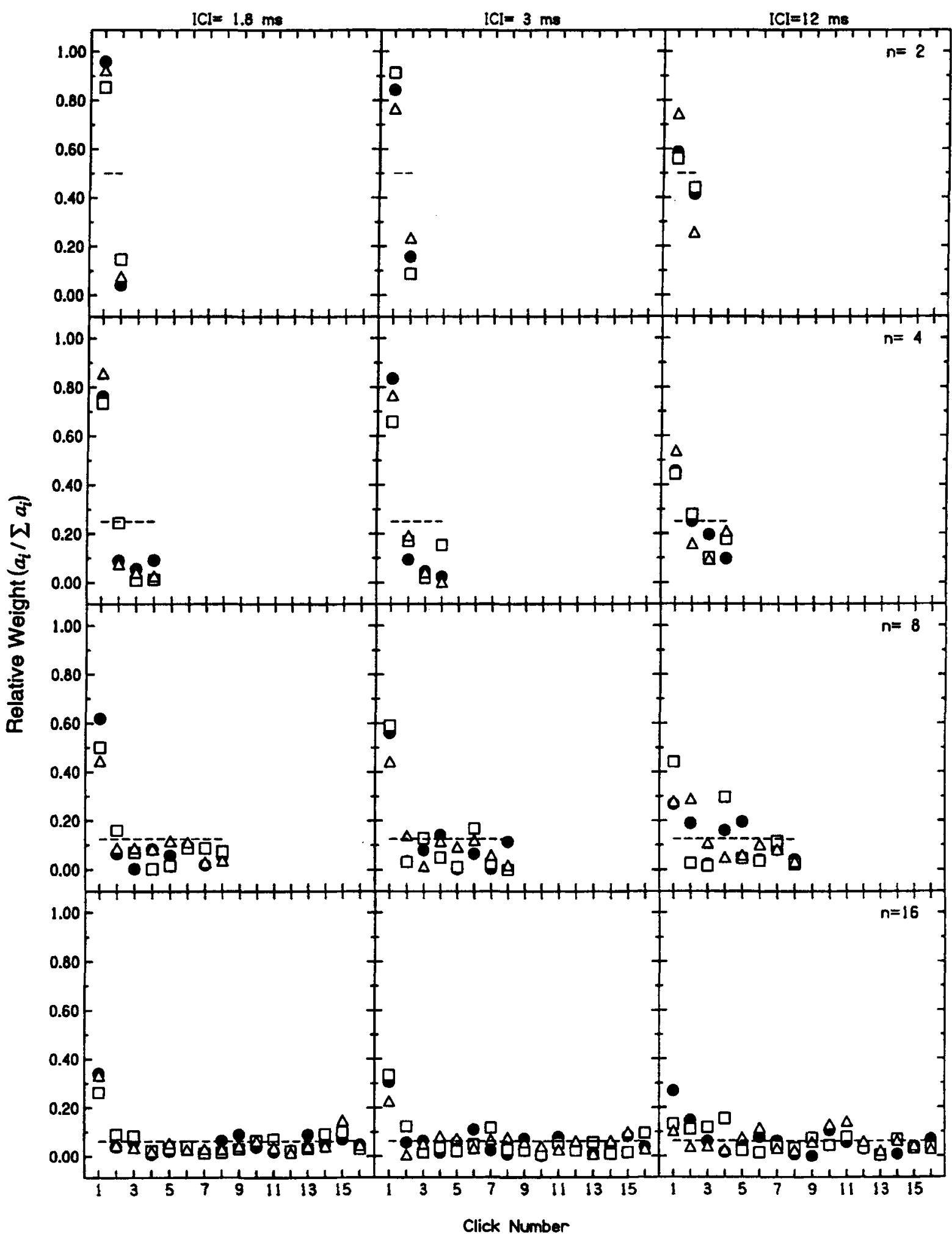

Figure 3. Observer weights determined for a train of $\boldsymbol{n}$ clicks. Each row shows weights for a different $\boldsymbol{n}$ ( 2 to 16). Each column shows weights for a different ICI $(1.8,3$, and $12 \mathrm{msec}$, respectively). Different symbols represent different observers (Y.B. $=$ circles; J.M. = squares; J.W. $=$ triangles). Dashed lines show equal (ideal) weightings $(1 / n)$. 


\section{Results}

Figure 3 shows individual results for 3 normal-hearing subjects $( \pm 10 \mathrm{~dB}$ ISO standards for frequencies between 250 and $10000 \mathrm{~Hz}$ ). Each column represents data for one ICI. Each row represents data for a fixed value of $n$. Dashed lines represent equal weightings $\left(n^{-1}\right)$. These lines represent ideal weight-distribution functions (Berg, 1990). Different symbols represent different subjects. Each point in each panel is based on a minimum of 1,600 trials -50 trials per each of 16 bins of each COSS function. Note, however, that the same 1,600 trials were used to obtain all weights within a given panel. The weights shown in this figure were based on a total of 540 different COSS functions.

Several points may be made regarding these weight distributions. Clearly, when the ICI is the smallest $(1.8 \mathrm{msec})$, the relative weight given to the onset click is greater than the weights given to other clicks. However, even though the relative onset weight becomes smaller as the ICI is increased, there is still a slightly greater weight given to the onset at the largest ICI of $12 \mathrm{msec}$. In the majority of cases, the weights given to the remaining clicks seem to be nearly the same, but unequal to zero even when ICI $=1.8 \mathrm{msec}$, suggesting that postonset information is, in fact, used in forming a decision [in some cases, e.g., $n=4$, the weight given to the 2nd click is statistically greater than the remaining postonset weights; $t(25)=3.00, p<.05$ ]. It is also worth noting that as the number of clicks in the train increases, a smaller proportion of total weight is given to the onset.

There are several ways of quantifying onset dominance, each revealing some important feature of this effect. One method of quantifying the dominance of onset information is to calculate the ratio of the weight given to the $1 \mathrm{st}$ click to the sum of weights given to subsequent clicks (we will refer to this ratio as the precedence ratio). These ratios, plotted in Figure 4, represent the relative influence of the onset and ongoing stimuli on the observer's decision making. A ratio of unity would imply that the onset and ongoing parts of the train were equally effective in influencing the observer's judgments. When the ICI is smallest and the number of clicks in the train is $n=2$, the onset has 10 times as much influence as the ongoing train, which in this case is only the 2 nd click. As the number of clicks increases, the onset carries progressively less influence in the decision. For trains of 8 and 16 clicks, the observer's judgments are more affected by the ongoing train than by the onset, even for the shortest ICI (a precedence ratio smaller than unity). Thus, the strength of precedence diminishes when more "echoes" are contained in the signal. An analysis of variance (ANOVA) on the data of Figure 3 showed significant effects on weight distribution caused by both the number of clicks $[F(3,35)=$ $6.58, p<.05]$ and ICI $[F(2,35)=5.00, p<.05]$. In two cases for which onset dominance was less obvious, ICI $=$ $12 \mathrm{msec}$ and $n=8$ and 16 , we performed a $t$ test between the means of onset and postonset weight distributions; in both cases, the onset weight carried a statistically significant greater weight $[t(23)=2.68, p<.05$, for $n=8$, and $t(46)=2.92, p<.05$, for $n=16]$.

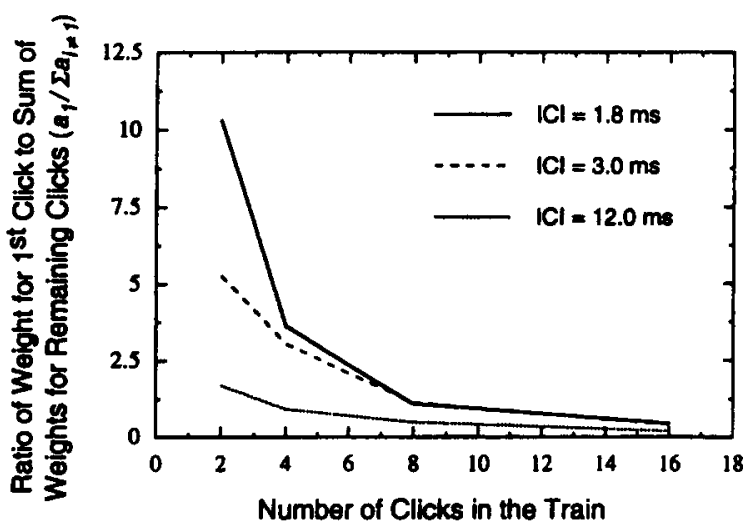

Figure 4. Precedence ratio. This is the ratio of the weight given to the 1 st click of the train to the sum of the remaining weights. Larger ratio represents greater onset dominance. A ratio of unity represents equal influence of the onset and ongoing train on the observer's decision. The parameter is ICI. Data are the average for 3 observers.

An alternative measure of onset dominance is the ratio of the weight given to the onset click to the average weight (instead of the sum) of the remaining clicks. The latter measure (which we will refer to as the average ratio) represents the degree to which the onset click is more influential than any other single click in determining the observer's judgments and may be an appropriate measure if postonset clicks have nearly the same weights. For comparison, the average ratios for an ICI of $1.8 \mathrm{msec}$ and $n=$ $2,4,8$, and 16 were $10.3,10.9,7.7$, and 6.8 , respectively. For $n=2$, this measure is identical to the precedence ratio. For $n=16$ and ICI $=1.8 \mathrm{msec}$, the onset carries, on the average, 6.8 times as much weight as a given postonset click of the train. Thus, while the influence of the onset in this case is reduced with increasing $n$, it is still more influential than the average postonset click.

\section{EXPERIMENT 2 \\ Weights Derived When Some Clicks Had Different Spectral Energies}

Hafter and Wenzel (1983) have suggested that onset dominance occurs within frequency channels. More recently, Blauert and Divenyi (1988) and Shinn-Cunningham, Zurek, Durlach, and Clifton (1995) have shown that when the onset has a lower frequency spectral contant than the trailing events, a strong onset dominance is observed, whereas when the trailing events have a lower frequency content than the onset, no onset dominance is observed. To better understand spectral effects for multicomponent stimuli, Experiment 2 was designed to measure weights given to each click as the frequency content of one or more clicks was altered relative to the remaining clicks.

\section{Method}

Three subjects were used in this experiment. One subject, J.M., had participated in Experiment 1; the other 2, C.S. and L.F., were new subjects. The stimuli were the same types as those used in Experiment 1 , with the following qualifications: The ICI was always 
$1.8 \mathrm{msec}$. The number of clicks in the train was 2,8 , or 16 . Most clicks had a carrier of $6 \mathrm{kHz}$; the others had a carrier of $3.5 \mathrm{kHz}$. The onset click always had a carrier of $6 \mathrm{kHz}$, so postonset clicks with the lower frequency content would be expected to be less influenced by onset dominance (Blauert \& Divenyi, 1988; ShinnCunningham et al., 1995). Bandwidths and click durations were the same as before. The standard deviation of the perturbations was also the same as that used in Experiment $l\left(\sigma_{i}=100 \mu \mathrm{sec}\right)$.

\section{Results}

Figure 5 shows averaged results for the 3 subjects. The points marked by an arrow represent clicks with a 3.5$\mathrm{kHz}$ carrier; otherwise, the carrier was $6 \mathrm{kHz}$ (unmarked points). In the top panel, weights are shown for a click train consisting of 2 clicks, both of which had carriers of $6 \mathrm{kHz}$. In the second panel, a 2-click train was used which was identical in every respect to the train used for the top panel except that the carrier of the 2 nd click was $3.5 \mathrm{kHz}$. The different weight distributions between the top and second panels suggests that when the 2 clicks have different spectral contents, onset dominance is nonexistent, denoting separate frequency channels. The third and fourth panels show results for $n \mathrm{~s}=8$ and 16 . What is evident from the latter two panels is that only the 1 st spectrally deviant click is heavily weighted. The remaining spectrally deviant clicks do not carry much weight and are not statistically different from other postonset clicks $[t(16)=$ n.s., for $n=8$ ]. When $n=16$, the mean weight of the 4 spectrally deviant clicks was statistically different from that of the remaining postonset clicks $[t(43)=1.85, p<$ $.05]$, with still a larger weight given to the 1 st spectrally deviant click when its mean weight is compared with the remaining nondeviant clicks $[t(34)=3.32, p<.05]$.

An important observation is the small weight given to the 8th click shown in the third panel $(n=8)$ of Figure 5 . As stated above, this weight is statistically no different from other nondeviant postonset weights. This was surprising, because the ICI between the 2 clicks marked by arrows is $7.2 \mathrm{msec}$ while the ICI between any 2 temporally adjacent clicks is $1.8 \mathrm{msec}$. It is possible that this may signify a between-frequency-channel influence. This observation and its implications are discussed in the discussion section.

\section{EXPERIMENT 3 \\ Weights Derived When There Were Gaps in the Train}

A "gap in the train" means that the ICI between 2 successive clicks is set to be longer than the ICI between other successive clicks. For example, in a train of 16 clicks, the ICI between successive clicks may be equal to $1.8 \mathrm{msec}$, with one exception-the ICI between Clicks 4 and 5 would be $5.4 \mathrm{msec}$. We refer to this different ICI as a "gap." Inserting such a temporal gap has been shown to improve the ability to lateralize a click train (Hafter \& Buell, 1990; Hafter et al., 1988). It is not clear, however, which clicks contribute to improved lateralization and what the distribution of weights is among individual clicks. Experiment 3 was conducted to measure these weights.

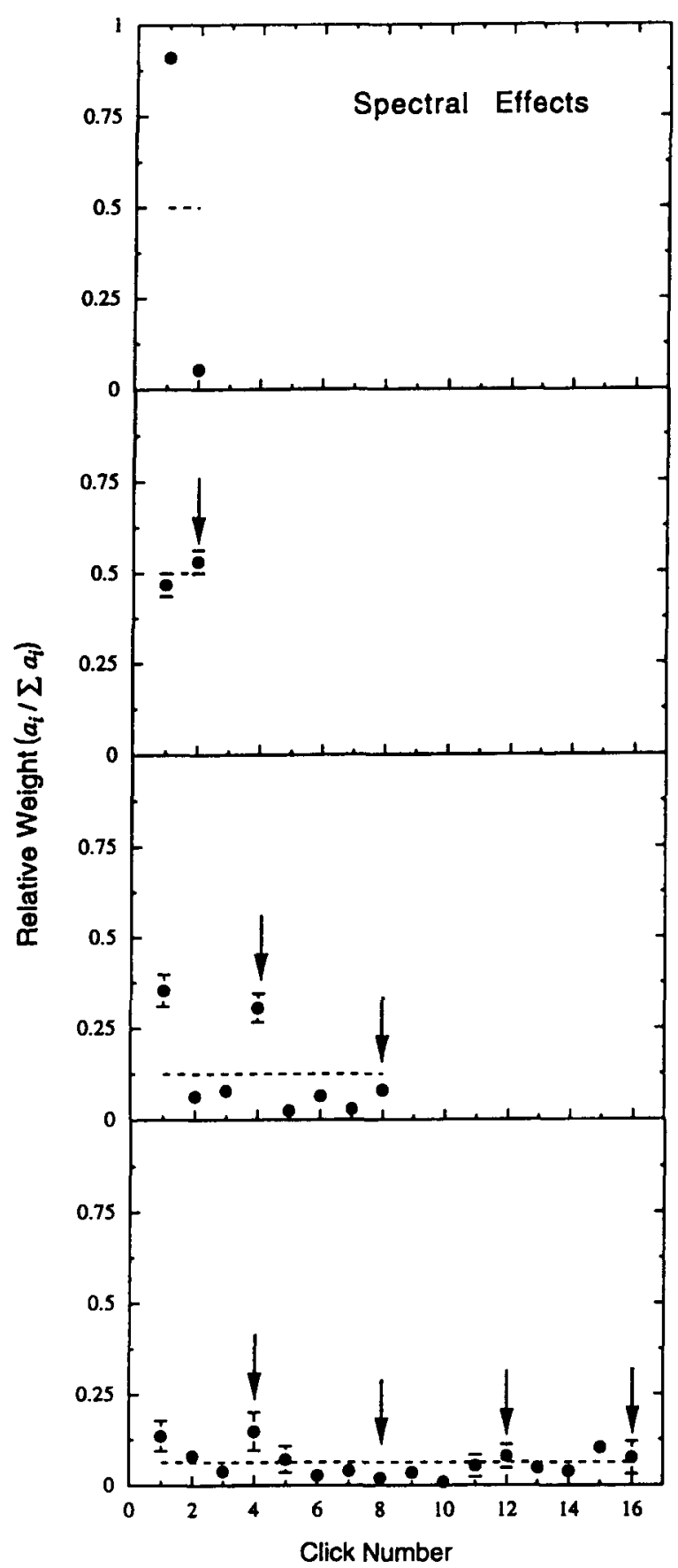

Figure 5. Spectral effects for trains of 2, 8, and 16 clicks. All clicks had a carrier of $6 \mathrm{kHz}$ unless marked by a vertical arrow. Click numbers marked by arrows had a carrier of $3.5 \mathrm{kHz}$. The ICI was $1.8 \mathrm{msec}$. Data are the average for 3 observers. Error bars are $1 S D$.

\section{Method}

The subjects were the same 3 who participated in Experiment 2. The train consisted of 16 clicks and the ICI was $1.8 \mathrm{msec}$. Three gaps were inserted in the train, dividing it into four segments of 4 clicks each. The gaps were $5.2 \mathrm{msec}$ in duration, and they replaced the normal ICI of $1.8 \mathrm{msec}$ where they were inserted. These gaps were inserted between Clicks 4-5, 8-9, and 12-13. 


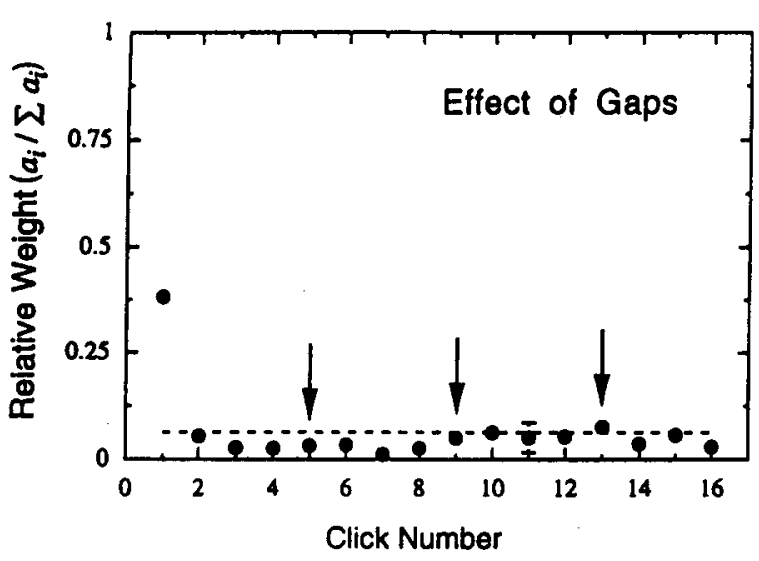

Figure 6. The effect of gaps on observer weighting for a train of 16 clicks. The ICIs between the click numbers marked by an arrow and the clicks immediately preceding them were $5.8 \mathrm{msec}$. The ICI between other successive clicks was $1.8 \mathrm{msec}$. Data are the average for 3 observers. Error bars are $1 S D$.

\section{Results}

Figure 6 shows the averaged results for the 3 subjects. The arrows mark the click that immediately followed the gap. This figure shows that inserting gaps was ineffectual $[t(43)=$ n.s. $]$. This result contrasts with that of Hafter et al. (1988), who reported that a gap at the center of a train of $n$ clicks improved performance by $\sqrt{2}$, compared with performance for $n / 2$ clicks. They suggested that such a gap "restarted" binaural processing, effectively reducing onset dominance. There is one important difference between the design used by Hafter et al. and that of the current study which may relate to this discrepancy. The ITD in that study was constant for all clicks, whereas in the current study it was a normal deviate. The randomly varying ITD may make it more difficult for the system to consider the gap as new information that would trigger a resampling of the stimulus.

\section{EXPERIMENT 4 \\ Weights Derived for a 3-Click Stimulus as a Function of the ICI Between the 1st and 2nd Clicks}

The purpose of Experiment 4 was to examine the function that relates onset dominance to ICI. Specifically, if 1 click was close enough in time to a 2nd click to dominate the lateral judgment, would there be a change in dominance if yet another click was introduced that preceded, in time, the original 2 clicks; that is, could the suppressor be suppressed as this newly introduced click was progressively brought closer in time to the original 2-click train?

\section{Method}

The subjects were the same 3 who participated in Experiment 2. We used a 3-click train in which the ICI between Clicks 2 and 3 was held constant at $1.8 \mathrm{msec}$, while the ICI between Clicks 1 and 2 $\left(\mathrm{ICI}_{1-2}\right)$ varied between 3.6 and $14.4 \mathrm{msec}$. Within a given run, the latter ICI was a constant. Weights were derived for all 3 clicks. It was expected that the 1 st of the 3 clicks would be given a large weight, consistent with previous observations. It was also expected that a substantial change in weight might be observed for the 2 nd and perhaps the 3 rd clicks as $\mathrm{ICI}_{1-2}$ varied. This, however, was not the case.

\section{Results}

Figure 7 shows averaged results for the 3 subjects. Two interesting outcomes are evident. First, the weight, $a_{2}$, given to the 2 nd click is small even for a large $\mathrm{ICI}_{1-2}$, and it is only slightly larger than $a_{3}$. Thus, even for very large $\mathrm{ICI}_{1-2}$, there is a strong onset dominance. This is surprising and not easily explained, because the data of Experiment 1 with a 2 -click train show that observers do give a substantial weight to the 2 nd click when the ICI is long. The presence of the 3 rd click in temporal proximity to the 2 nd reduces the weight given to the latter click. A second outcome is that the weights are related to ICI by a linear function, $a_{i}=a_{0}+m \mathrm{ICI}$, where the slope, $m$, is negative for $a_{1}$ and positive otherwise. This slope reflects the effect of changing the value of $\mathrm{ICI}_{1-2}$ with larger $m$ corresponding to a larger effect. The slope is generally small ( $m=-0.015,0.009$, and 0.006 for $a_{1}, a_{2}$, and $a_{3}$, respectively). The difference between the slope of the fits for $a_{2}$ and $a_{3}$ approaches significance $[t(4)=$ $1.86, p<.1]$. If, at a large $\mathrm{ICI}_{1-2}$, the 2 nd click was given a large weight equivalent to that given to the 1 st click, then we would have expected a function for $a_{2}$ which would have terminated at a relative weight of at least $1 / 3$. The latter expected function has a slope of 0.027 , three times larger than the observed slope.

The linear fits are very good, with over $98 \%$ of the total variance accounted for in all cases $\left(r^{2}=0.989,0.995\right.$, and 0.991 for $a_{1}, a_{2}$, and $a_{3}$, respectively). Interestingly, the $y$-intercept approaches unity $(0.989)$ for the fit to $a_{1}$, and it approaches zero for $a_{2}$ and $a_{3}(0.009$ and 0.002 , respectively), suggesting a total dominance of onset at ICI $=0$. Empirical evidence, however, suggests otherwise; reports have shown that as ICI approaches values smaller than $1 \mathrm{msec}$, the weight given to the 2 nd click of a train actually

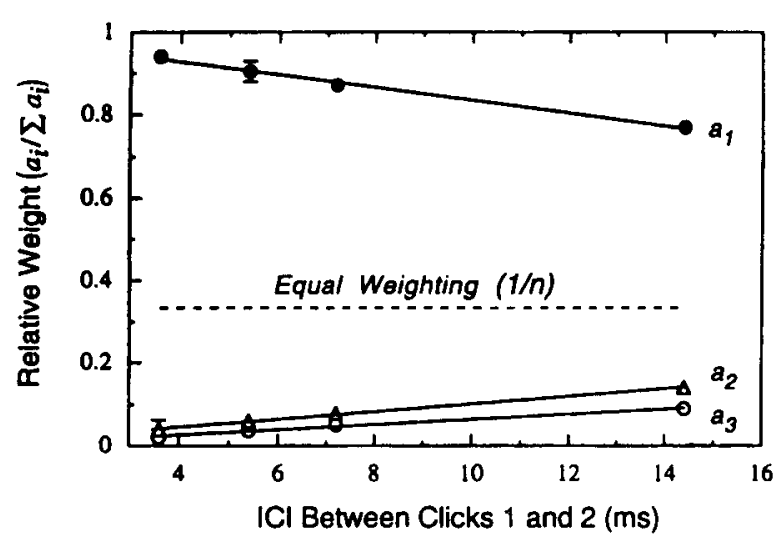

Figure 7. Observer weightings for a train of 3 clicks. The ICI between Clicks 2 and 3 was fixed at $1.8 \mathrm{msec}$. The ICI between Clicks 1 and 2 varied between runs and ranged from 3.6 to 14.4 msec. $a_{1}$ to $a_{3}$ represent weights estimated for Clicks $1-3$, respectively. Data are the average for 3 observers. Error bars are $1 S D$. 
increases, an effect that has been referred to as summing localization and denoting a secondary mechanism (Blauert, 1983; Zurek, 1980, 1987). Therefore, one should be cautious in interpolations for very brief ICIs due to this nonlinear deviation of weights as ICI approaches zero.

\section{DISCUSSION \\ What Do Weighting Functions Reveal About Onset Dominance?}

Weight analysis is a general technique with wide applications. The data of Experiment 1 (Figure 3), which are in line with previous work, show that the technique may be applied to binaural paradigms. A few novel results are noteworthy. Figure 3 shows that, for most cases, postonset clicks carry nearly equal weights and there are no significant differences between weight estimates for earlier versus later arriving clicks after the 1 st (e.g., no memorybased recency or offset effect as seen with other psychoacoustic phenomena; Berg, 1989; Watson et al., 1966). In some cases, however, the 2 nd click of the train is given a statistically greater weight than the remaining clicks $[n=4$ in Figure $3 ; t(25)=3.00, p<.05]$; for $n>4$, postonset weights do not differ significantly.

Another observation is that relative onset weights diminish with increasing $n$ and ICI. For a given $n$, it may be advantageous to reduce reliance on one component (onset) if such strategy improves signal detection. Consider, for example, the simple case where $n=2$ and ICI is reasonably long (upper-right panel of Figure 3 ). In the general case, $d^{\prime}$, the index of detectability, may be written as

$$
d^{\prime}=\frac{\sum_{\text {All } i} a_{i} E\left[\operatorname{ITD}_{i}+\varepsilon_{i}\right]}{\sqrt{\sum_{\text {All } i} a_{i}^{2}\left(\sigma_{i}^{2}+\sigma_{\varepsilon_{i}}^{2}\right)}},
$$

where $\varepsilon_{i} \sim$ normal $\left(0, \sigma_{\varepsilon_{i}}^{2}\right)$ represents internal noise. Since $E\left(\mathrm{ITD}_{i}+\varepsilon_{i}\right), \sigma_{i}$, and $\sum a_{i}$ are constant, if we assume $\sigma_{\varepsilon_{i}}=\sigma_{\varepsilon_{j \neq i}}$ or $\sigma_{\varepsilon_{i}} \ll \sigma_{i}$, then

$$
d^{\prime} \alpha 1 / \sqrt{ } \sum a_{i}^{2}
$$

If $\sum a_{i}=1$, how should $a_{i}$ be selected to optimize $d^{\prime}$ ? Maximizing $d^{\prime}$ requires that weights be distributed equally across all $a_{i}$ (Berg, 1990); the optimum weights would be $a_{1}=a_{2}=0.5 . d^{\prime}$ is at a minimum when the entire weight is given to one component, for example, $a_{1}=1, a_{2}=0$ (a $d^{\prime}$ factor of $\sqrt{ } 2$ difference). Thus, the relative reduction in the weight given to Click 1 in the upper-right panel of Figure 3 (compared with the upper left panel) is consistent with optimizing signal detection.

The weighting functions also suggest that onset dominance is only partially a within-frequency-channel effect (see Figure 5). Only the 1st spectrally deviant click is weighted greater than other postonset clicks. The 2 nd, 3rd, or 4th deviant clicks seem to carry about as much weight as nondeviant, postonset clicks. That onset dominance occurs only partially within channels is suggested by the third panel of Figure 5. The ICI between succes- sive clicks, irrespective of their spectral content, is $1.8 \mathrm{msec}$. The ICI between the 2 spectrally deviant clicks (arrows) is $7.2 \mathrm{msec}$, yet the weight given to Click 8 is about the same as the weights for other postonset clicks $[t(16)=0.38$, n.s.]. This is surprising, because if frequency channels were truly independent, then an ICI of $7.2 \mathrm{msec}$ should result in a greater weight for Click 8 . Although we have not directly compared ICIs of 1.8 and $7.2 \mathrm{msec}$, previous studies using impulsive stimuli have shown large differences between ICIs whose magnitudes are within the bounds used here (ICI 2 vs. $5 \mathrm{msec}$; Hafter et al., 1988, Figure 4). That Click 8 does not receive a relatively larger weight suggests that onset dominance occurs only partially within frequency channels. Other factors that can influence weightings across frequency include cognitive load or cross-channel binaural interference (Buell \& Trahiotis, 1993; Dye, 1990; McFadden \& Pasanen, 1976; Shinn-Cunningham et al., 1995; Trahiotis \& Bernstein, 1990; Zurek, 1985).

Weight estimates for individual clicks also provide a more detailed view of how nonuniform ICIs affect onset dominance (Figures 6 and 7). A surprising result was the small weight given to Click 2 of a 3 -click train, even when the ICI between Clicks 1 and 2 was long (Figure 7). This finding has interesting implications. In addition to what precedes a given click, what occurs after this click also affects its contribution to the weight distributions. For a 2-click train, an ICI of 14.4 msec would seem sufficient to yield nearly equal weights for Clicks 1 and 2 (see Figure 3, top-right panel for ICI $=12 \mathrm{msec}$ ). However, adding a 3rd click, $1.8 \mathrm{msec}$ after Click 2 , reduces the weight given to Click 2 . These results suggest that the train may be processed as one entity; the decision is based not only on each discrete component but also on the overall stimulus design.

\section{Observer Efficiency}

Performance of an observer may be compared with that achieved by an ideal observer who optimally distributes weights among components. When all components have equal perturbations $\left(\sigma_{1}=\sigma_{2}=\ldots=\sigma_{n}\right)$ with reasonable assumptions about additive internal noise, ideal weights may be written as $\hat{a}_{i}=n^{-1}$. To facilitate comparison between conditions where the observed $d$ 's are different, Berg (1990) has derived the efficiency of an observer in distributing weights among components by using Tanner and Birdsall's (1958) definition

$$
\eta=\left(d_{\text {obs }}^{\prime} / d_{\text {ideal }}^{\prime}\right)^{2}
$$

in which one can substitute Equation 3 and simplify for the equal-variance case to obtain

$$
\eta=\sum \hat{a}_{i}^{2} / \sum a_{i}^{2}
$$

Figure 8 plots $\eta$ for the 12 conditions of Figure 3. Observers are able to distribute weights more efficiently for the longer ICI, $12 \mathrm{msec}$, but there is no substantial difference between efficiencies for ICIs of 3 and $1.8 \mathrm{msec}$. Observer efficiency in distributing weights is greater when 


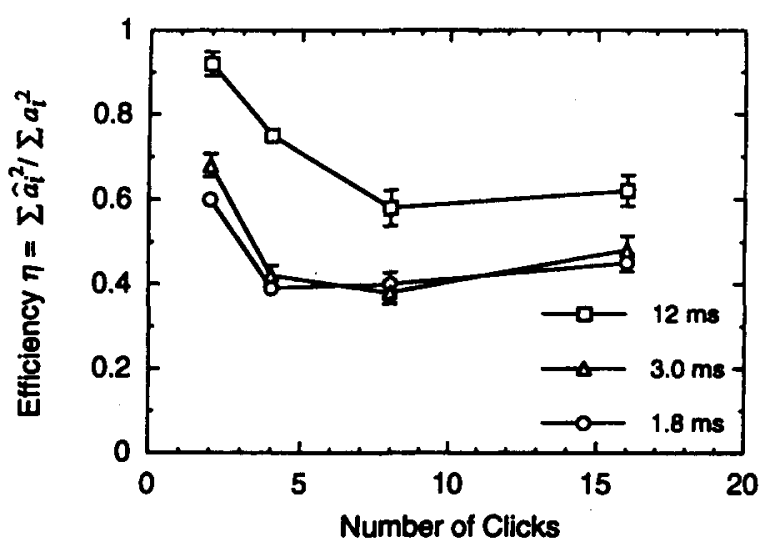

Figure 8. Efficiency of weight distribution for data of Figure 3, averaged across 3 observers.

$n=2$, approaching near-perfect efficiency when ICI is $12 \mathrm{msec}$. Because ideal weighting requires that the weights be distributed equally among components, as the onset weight increases, the denominator in Equation 6 will approach its maximum value, unity; for large $n$, this produces very inefficient weight distributions. For the shorter ICIs, the efficiency seems to have reached a lower asymptote when $n$ is greater than 2 . What is the lowest possible efficiency for a given $n$ ? For a specified $n$, one may consider $\eta$ when $\sum a_{i}=1$ is entirely allocated to the 1 st click. This is the least efficient weight-distribution strategy. Consider efficiency for ICI $=1.8 \mathrm{msec}$ and $n=8$; this condition produces one of the least efficient observer performances $(\eta=0.4)$. For this condition, the lower bound efficiency is 0.35 , and the observed $\eta$ is only slightly better than least efficient performance.

\section{Deficiencies in Explanations Based on Cross-Correlation Analysis and \\ Lindemann's Contralateral Inhibition}

It is appropriate to end with a word on current theories of binaural interaction. It is becoming increasingly clear that recent findings on onset dominance cannot be satisfactorily explained with models of cross-correlation. These models simulate neural processing at the peripheral auditory tract up to the nuclei of the superior olivery complex and the colliculus. They are designed for relatively short time constants, as expected from current understanding of the auditory periphery. Some cross-correlation models have attempted to predict empirical observations related to onset dominance. One popular model described by Lindemann (1986a, 1986b) uses a contralateral inhibitory network; the inhibition is a multiplier whose magnitude is a function of cross-correlation delay and a decaying function of running time. Its popularity stems from its physiologically realistic design (lateral inhibition) and its ability to explain simple observations such as the lateralization of clicks with ICI smaller than the "echo threshold" (Blauert, 1983) and the lateralization of single postonset clicks.
This model and others (e.g., Zurek, 1987) that include an explicit inhibitory mechanism cannot easily explain results that necessitate long time constants. The Clifton effect (1987) and the Franssen effect (1960) are two examples that seem to involve time constants on the order of several seconds, clearly beyond the computational capabilities of peripheral structures and those assumed in these models. Other recent findings, which do not require long time constants, are still difficult to predict with crosscorrelation analysis or simple inhibitory networks. For example, Lindemann's (1986a, 1986b) model cannot account for results of our experiment on 3-click trains (Figure 7). In a 2-click train with a long ICI, the 2nd click would carry substantial spatial information. However, if a 3 rd click is added with a short ICI ( $1.8 \mathrm{msec})$, the 2 nd click now becomes much less informative. Contralateral inhibition, which may explain onset dominance with a 2-click train, cannot explain this finding with 3 clicks. The results of this 3-click experiment also seem to be in contrast with the assumption made by Houtgast and Aoki's (1994) weight-estimation method that later occurring events do not influence earlier events in an onset-dominance paradigm.

Other studies also lend support to deficiencies in simple inhibitory models. Hafter et al. (1988, p. 670) suggested that Lindemann's contralateral inhibition would have difficulty in explaining active "restarting" in onset dominance; they show that triggers that signify new information may cause resampling of the spatial environment by the binaural system, thereby releasing the posttrigger clicks from the effects of the dominating 1 st click. This suggests that onset dominance may involve active neural mechanisms. In agreement with these observations, Saberi and Perrott (1995) have shown that a single onset click can affect the perceived lateral position of an entire train composed of 200 clicks and lasting hundreds of milliseconds. It is difficult to see how simple neural inhibition can last for such long periods. Other factors should, therefore, be taken into account. These may include perceptual grouping and attentional factors, plausibility of stimulus features (Clifton, 1987; Hartmann \& Rakerd, 1989), temporal sluggishness (Grantham \& Wightman, 1978), active release from suppression (Hafter et al., 1988), and limits on sensory or cognitive resources.

\section{REFERENCES}

Aoki, S., \& Houtgast, T. (1992). A precedence effect in the perception of interaural cross correlation. Hearing Research, 59, 25-30.

BERG, B. G. (1989). Analysis of weights in multiple observation tasks. Journal of the Acoustical Society of America, 86, 1743-1746.

BERG, B. G. (1990). Observer efficiency and weights in a multiple observation task. Journal of the Acoustical Society of America, 88, 149-158.

BlauerT, J. P. (1983). Spatial hearing. Cambridge, MA: MIT Press. BlauerT, J. P. (1989). Binaural technology: Fundamentals and applications. Journal of the Acoustical Society of America, 86, S66.

BlaUeRT, J., \& COL, J. (1991). Irregularities in the precedence effect. In Y. Cazals, L. Demany, \& K. Horner (Eds.), Auditory physiology and perception: Proceedings of the 9 th International Symposium on Hearing (pp. 531-538). Oxford, U.K.: Pergamon Press. 
Blauert, J., \& Divenyı, P. L. (1988). Spectral selectivity in binaural contralateral inhibition. Acustica, 66, 267-274.

Buell, T. N., \& Trahiotis, T. (1993). Interaural temporal discrimination using two sinusoidally amplitude-modulated, high-frequency tones: Conditions of summation and interference. Journal of the Acoustical Society of America, 93, 480-487.

Clifton, R. K. (1987). Breakdown of echo suppression in the precedence effect. Journal of the Acoustical Society of America, 82, 1834-1835.

Cranford, J., \& Oberholtzer, M. (1976). Role of neocortex in binaural hearing in the cat: II. The "precedence effect" in sound localization. Brain Research, 111, 225-239.

DomnITZ, R. H. (1973). A headphone monitoring system for binaural experiments below $1 \mathrm{kHz}$. Journal of the Acoustical Society of America, 58, 510-511.

DYE, R. H., JR. (1990). The combination of interaural information across frequencies: Lateralization on the basis of interaural delay. Journal of the Acoustical Society of America, 88, 2159-2170.

FransSEN, N. V. (1960). Some considerations on the mechanism of directional hearing. Unpublished doctoral dissertation, Technische Hogeschool, Delft, The Netherlands.

GaSkEll, H. (1983). The precedence effect. Hearing Research, 11, 277-303.

Grantham, D. W., \& Wightman, F. L. (1978). Detectability of varying interaural temporal differences. Journal of the Acoustical Society of America, 63, $511-523$.

HAAS, H. (1972). The influence of a single echo on the audibility of speech (K. P. R. Ehrenberg, Trans.). Journal of the Audio Engineering Society, 20, 146-159. (Translation from Über den Einfluss des Einfachechos auf die Hörsamkeit von Sprache. Unpublished doctoral dissertation, University of Göttingen, Germany, 1949.)

HAFTER, E. R., \& Buell, T. N. (1990). Restarting the adapted binaural system. Journal of the Acoustical Society of America, 88, 806-812.

HAFTER, E. R., BuELl, T. N., \& RichaRds, V. M. (1988). Onset coding in lateralization: Its form, site, and function. In G. M. Edelman, W. E. Gall, \& W. M. Cowan (Eds.), Auditory function (pp. 647-676). New York: Wiley.

HAFTER, E. R., \& DYE, R. H. (1983). Detection of interaural differences of time in trains of high-frequency clicks as a function of interclick interval and number. Journal of the Acoustical Society of America, 73, 1708-1713.

HAFTER, E. R., \& WENZEL, E. M. (1983). Lateralization of transients presented at high rates: Site of the saturation effect. In R. Klinke \& R. Hartmann (Eds.), Hearing: Physiological basis and psychophysics (pp. 202-208). Berlin: Springer-Verlag.

HaRTMANN, W. M., \& RAKERD, B. (1989). Localization of sound in rooms: IV. The Franssen effect. Journal of the Acoustical Society of America, 86, 1366-1373.

HenRY, J. (1851). [Presentation before the American Association for the Advancement of Science on August 21, 1849]. In Scientific writings of Joseph Henry: Part II, 1847-1878 (pp. 295-296). Washington, DC: Smithsonian Institution.

Hochster, M. E., \& Kelly, J. B. (1981). The precedence effect and sound localization by children with temporal lobe epilepsy. Neuropsychologia, 19, 49-55.

HoutGast, T., \& AOKI, S. (1994). Stimulus-onset dominance in the perception of binaural information. Hearing Research, 72, 29-36.

LINDEMANN, W. (1986a). Extension of a binaural cross-correlation model by contralateral inhibition: I. Simulation of lateralization for stationary signals. Journal of the Acoustical Society of America, 80, 1608-1622.

LINDEMANN, W. (1986b). Extension of a binaural cross-correlation model by contralateral inhibition: II. The law of the first wavefront. Journal of the Acoustical Society of America, 80, 1623-1630.

LJTovsky, R. Y. (1994). Evidence for physiological correlates of the precedence effect in the inferior colliculus of kittens. Journal of the Acoustical Society of America, 95(Suppl. 1), 2843.

Litovsky, R. Y., \& Macmillan, N. A. (1994). Sound localization precision under conditions of the precedence effect: Effects of azimuth and standard stimuli. Journal of the Acoustical Society of America, 96, 752-758.
McFadden, D., Pasanen, E. G. (1976). Lateralization at high frequencies based on interaural time differences. Journal of the Acoustical Society of America, 59, 634-639.

Muncey, R. W., Nickson, A. F. B., \& Dubout, P. (1953). The acceptability of speech and music with a single artificial echo. Acustica, $\mathbf{3}$, 168-173.

Perrott, D. R., Marlborough, K., Merrill, P., \& Strybel, T. Z. (1989). Minimum audible angle thresholds obtained under conditions in which the precedence effect is assumed to operate. Journal of the Acoustical Society of America, 85, 282-288.

RichaRDS, V. M., \& ZHU, S. (1994). Relative estimates of combination weights, decision criteria, and internal noise based on correlation coefficients. Journal of the Acoustical Society of America, 95, 423-434.

SABERI, K., \& Perrotr, D. R. (1990). Lateralization thresholds obtained under conditions in which the precedence effect is assumed to operate. Journal of the Acoustical Society of America, 87, 1732-1737.

SABERI, K., \& PERROTT, D. R. (1995). Lateralization of click-trains with opposing onset and ongoing interaural delays. Acustica, 81, 272-275.

Shinn-Cunningham, B. G., Zurek, P. M., \& Durlach, N. I. (1993). Adjustment and discrimination measurements of the precedence effect. Journal of the Acoustical Society of America, 93, 2923-2932.

Shinn-Cunningham, B. G., Zurek, P. M., Durlach, N. I., \& Clifton, R. K. (1995). Cross-frequency interactions in the precedence effect. Journal of the Acoustical Society of America, 98, 164-171.

SNOw, W. B. (1954). Effect of arrival time on stereophonic localization. Journal of the Acoustical Society of America, 26, 1071-1074.

TANneR, W. P., \& Birdsall, T. G. (1958). Definitions of $d^{\prime}$ and $\eta$ as psychophysical measures. Journal of the Acoustical Society of America, 30, 922-928.

TOBIAS, J. V., \& SchUBERT, E. D. (1959). Effective onset duration of auditory stimuli. Journal of the Acoustical Society of America, 31, 1595-1605.

Trahiotis, T., \& Bernstein, L. R. (1990). Detectability of interaural delays over select spectral regions: Effects of flanking noise. Journal of the Acoustical Society of America, 87, 810-813.

Wallach, H., Newman, E. B., \& Rosenzweig, M. R. (1949). The precedence effect in sound localization. American Journal of Psychology, 52, 315-336.

Watson, C. S., Wroton, H. W., Kelly, W. J., \& Benbassat, C. A. (1966). Factors in discrimination of tonal patterns: I. Component frequency, temporal position and silent intervals. Journal of the Acoustical Society of America, 57, 1175-1185.

YIN, T. C. T., \& LITovsKY, R. Y. (1994). Physiological studies of the precedence effect in the inferior colliculus of the cat. In Advances in Hearing Research: Proceedings of the 10th International Symposium on Hearing, Irsee, Bavaria. River Edge, NJ: World Scientific.

Yost, W. A., \& Soderquist, D. R. (1984). The precedence effect: Revisited. Journal of the Acoustical Society of America, 76, 1377-1383.

ZUREK, P. M. (1980). The precedence effect and its possible role in the avoidance of interaural ambiguities. Journal of the Acoustical Society of America, 67, 952-964.

ZuREK, P. M. (1985). Spectral dominance in sensitivity to interaural delay for broadband stimuli. Journal of the Acoustical Society of America, 78(Suppl. 1), S18.

ZuREK, P. M. (1987). The precedence effect. In W. A. Yost \& G. Gourevitch (Eds.), Directional hearing (pp. 85-105). New York: SpringerVerlag.

\section{NOTE}

1. A related method of estimating relative weights has recently been suggested by Richards and Zhu (1994). Like Berg's procedure, the value of each component is a normal deviate; however, they calculate the Pearson product-moment correlation coefficient between an observer's responses and the perturbation values of each component.

(Manuscript received October 31, 1994; revision accepted for publication January 14, 1996.) 